\title{
Some New Integral Inequalities Related to Convex Functions
}

\author{
Çetin YILDIZ ${ }^{1}$, Mustafa GÜRBÜZ²
}

ABSTRACT: We gathered several new integral inequalities with the help of a kernel and using functions which are convex. The proofs are made with quite basic analysis and stated on Hölder and Power mean inequalities.

Keywords: convexity, convex functions, integral inequality

\section{Konveks Fonksiyonlarla İlgili Bazı Yeni İntegral Eşitsizlikler}

ÖZET: Konveks fonksiyonları kullanarak, bir çekirdek yardımıyla bazı yeni integral eşitsizlikler elde ettik. İspatlarda kullanılan analiz oldukça elementer olup Hölder ve Power mean eşitsizliği üzerine kurgulandı.

Anahtar Kelimeler: konvekslik, integral eşitsizlik, konveks fonksiyonlar

Çetin YILDIZ (0000-0002-8302-343X), Atatürk University, Kazım Karabekir Education Faculty, Mathematics Department, Erzurum, Türkiye

Mustafa GÜRBÜZ (0000-0002-7092-4298), Ağrı İbrahim Çeçen University, Education Faculty, Mathematics Department, Ağrı, Türkiye Sorumlu yazar/Corresponding Author: Mustafa GÜRBÜZ, mgurbuz@agri.edu.tr

Bu çalışma 18-21 Nisan 2017 tarihinde Antalya'da düzenlenen "International Conference on Advances in Natural and Applied Sciences" kongresinde sunulmuş ve kongre özet kitabında yayınlanmışıtır. 


\section{INTRODUCTION}

Convexity, with very old background, has many application areas such as probability theory, graph theory, functional analysis and also nonlinear analysis. Inequalities have application areas not only in mathematics but also in physics and

$$
f\left(\frac{a+b}{2}\right) \leq \frac{1}{b-a} \int_{a}^{b} f(x) d x \leq \frac{f(a)+f(b)}{2}
$$

holds where $a, b \in I \subseteq \mathbb{R}$ with $a$ is less than or equal to $b$. That is, image of midpoint of bounds of domain is always less than or equal to mean value of function and mean value of function is less than or equal to midpoint of images of bounds of domain. Many papers including Hermite-Hadamard, Ostrowski, Simpson etc. type results valid for engineering. Hermite-Hadamard inequality is very significant inequality in the literature which was published in Mathesis 3 in 1883. This famous inequality says if $f$ is a convex mapping defined from $I$ to $\mathbb{R}$ then

different types of convex functions classes have been published. Some of those inequalities have been put together in a monograph in Dragomir and Pearce (2000) which is very useful for interested readers. On the other hand, there are many generalizations of this inequality with fractional integral types.

\section{MATERIAL AND METHOD}

In 1984, Toader introduced the $m$-convexity definition (1984, as cited in Dragomir and Pearce, 2000) as noted below:

Definition 2.1. "The function $f:[0, b] \rightarrow \mathbb{R}$ is said to be $m$-convex, where $m \in[0,1]$, if for every $x, y \in[0, b]$ and $t \in[0,1]$ we have:

$f(t x+m(1-t) y) \leq t f(x)+m(1-t) f(y)$.

Denote by $K_{m}(b)$ the set of the $m$-convex functions on $[0, b]$ for which $f(0) \leq 0$ " (p.293).

For $m=1$, definition of $m$-convex functions reduces to definition of classical convex 
functions and also for $m=0$ definition of $m$-convex functions reduces to definition of starshaped functions defined on same domain with $m$-convex function. Also some new results has been proved by Lara et al. (2017).

Example 2.1. Let $x \in[0, r]$ with $r>0, f$ defined from $[0, r]$ to $\mathbb{R}$ be a linear function described as $f(x)=m x+n(m, n \in \mathbb{R})$ with $a \neq 0$. If $n \leq 0$, then we have $f \in K_{m}(r)$ (Gürbüz, 2013).

Besides, Miheşan (1993) introduced $(\alpha, m)$ - convexity (1993, as cited in Dragomir and Pearce, 2000) as noted below:

Definition 2.2. "The function $f:[0, b] \rightarrow \mathbb{R}$ is said to be $(\alpha, m)$-convex, where $(\alpha, m) \in$ $[0,1]^{2}$, if for every $x, y \in[0, b]$ and $t \in[0,1]$ we have $f(t x+m(1-t) y) \leq t^{\alpha} f(x)+m\left(1-t^{\alpha}\right) f(y)$.

Note that for $(\alpha, m) \in\{(0,0),(\alpha, 0),(1,0),(1, m),(1,1),(\alpha, 1)\}$, one obtains the following classes of functions: increasing, $\alpha$-starshaped, starshaped, $m$-convex, convex and $\alpha$-convex. Denote by $K_{m}^{\alpha}(b)$ the set of the $(\alpha, m)$-convex functions on $[0, b]$ for which $f(0) \leq 0 "($ p.299)

In our main results we came across with Euler's integral of the first kind which is also well known as Beta function which is defined as:

$$
\beta(x, y)=\int_{0}^{1} t^{x}(1-t)^{y-1} d t
$$

for $x, y>0$ (Chaudhry, 1997).

To find more information about convexity in the mean of partial ordering, one can see the references of Bakula et al., 2008; Özdemir et al., 2010; Özdemir et al., 2011; Alomari et al., 2012; İşcan, 2013; Y1ldız et al. 2013; Set et al., 2017.

We used the following lemma gathered by Y1ldiz (2018) for evidence of our theorems:

Lemma 2.1. "Let $f:[a, b] \rightarrow \mathbb{R}$ be $n$-times differentiable function. If $f^{(n)} \in L[a, b]$, then 


$$
\begin{aligned}
\int_{a}^{b} f(t) d t=\sum_{k=0}^{n-1} \frac{f^{(k)}(a)+(-1)^{k} f^{(k)}(b)}{(k+1) !}\left(\frac{b-a}{2}\right)^{k+1} & \\
+(-1)^{n} \frac{(b-a)^{n+1}}{2^{n+1} n !} & \left\{\int_{0}^{1}(t-1)^{n} f^{(n)}\left(t \frac{a+b}{2}+(1-t) a\right) d t\right. \\
& \left.+\int_{0}^{1}(1-t)^{n} f^{(n)}\left(t \frac{a+b}{2}+(1-t) b\right) d t\right\}
\end{aligned}
$$

where $n$ is a naturel number with $n \geq 1$ " (p.2)

The primary aim of the current study is to handle some new and original inequalities handled by using $(\alpha, m)$-convex functions. Results are associated with the thousands of times cited integral inequality by name Hermite-Hadamard. We will typify the notation $\mathfrak{R}$ instead of “( $\left(\int_{a}^{b} f(t) d t-\sum_{k=0}^{n-1} \frac{f^{(k)}(a)+(-1)^{k} f^{(k)}(b)}{(k+1) !}\left(\frac{b-a}{2}\right)^{k+1}\right)$ " throughout the paper.

\section{RESULTS AND DISCUSSIONS}

In present part, we handled some new results for $(\alpha, m)$-convex function by using Hölder and Power-mean inequalities. Also with special selections of $\alpha$ and $m$, some new results are revealed.

Theorem 3.1. Let $f$ defined from $[0, b]$ to $\mathbb{R}$ be $n$-times differentiable $(\alpha, m)$-convex function. If $f^{(n)} \in L[a, b]$ with $0<a<b$ then

$$
\begin{aligned}
|\Re| \leq \frac{(b-a)^{1+n}}{2^{1+n} n !}\{ & 2 \beta(1+n, 1+\alpha)\left|f^{(n)}\left(\frac{b+a}{2}\right)\right| \\
& \left.+m\left(\frac{1}{1+n}-\beta(1+n, 1+\alpha)\right)\left[\left|f^{(n)}\left(\frac{a}{m}\right)\right|+\left|f^{(n)}\left(\frac{b}{m}\right)\right|\right]\right\}
\end{aligned}
$$

where $(\alpha, m) \in[0,1] \times[0,1], n$ naturel number, $n \geq 1$ and $\beta(.,$.$) is used to specify$ Euler's integral of the first kind.

Proof. By means of Lemma 2.1. and features of absolute value we get

$$
\begin{aligned}
|\Re| \leq \frac{(b-a)^{1+n}}{2^{1+n} n !}\{ & \left\{\int_{0}^{1}(1-t)^{n}\left|f^{(n)}\left(t \frac{b+a}{2}+m(1-t) \frac{a}{m}\right)\right| d t\right. \\
& \left.+\int_{0}^{1}(1-t)^{n}\left|f^{(n)}\left(t \frac{b+a}{2}+m(1-t) \frac{b}{m}\right)\right| d t\right\} .
\end{aligned}
$$


With the help of $(\alpha, m)$-convexity of $\left|f^{(n)}\right|$ we note that

$$
\begin{aligned}
|\Re| \leq & \frac{(b-a)^{1+n}}{2^{1+n} n !}\left\{\int_{0}^{1}(1-t)^{n}\left[t^{\alpha}\left|f^{(n)}\left(\frac{b+a}{2}\right)\right|+m\left(1-t^{\alpha}\right)\left|f^{(n)}\left(\frac{a}{m}\right)\right|\right] d t\right. \\
& \left.+\int_{0}^{1}(1-t)^{n}\left[t^{\alpha}\left|f^{(n)}\left(\frac{b+a}{2}\right)\right|+m\left(1-t^{\alpha}\right)\left|f^{(n)}\left(\frac{b}{m}\right)\right|\right] d t\right\} \\
= & \frac{(b-a)^{1+n}}{2^{1+n} n !} \\
& \times\left\{\beta(1+\alpha, 1+n)\left|f^{(n)}\left(\frac{b+a}{2}\right)\right|+m\left(\frac{1}{1+n}-\beta(1+n, 1+\alpha)\right)\left|f^{(n)}\left(\frac{a}{m}\right)\right|\right. \\
& \left.+\beta(1+\alpha, 1+n)\left|f^{(n)}\left(\frac{b+a}{2}\right)\right|+m\left(\frac{1}{1+n}-\beta(1+n, 1+\alpha)\right)\left|f^{(n)}\left(\frac{b}{m}\right)\right|\right\} \\
= & \frac{(b-a)^{1+n}}{2^{1+n} n !}\left\{2 \beta(1+n, 1+\alpha)\left|f^{(n)}\left(\frac{b+a}{2}\right)\right|\right. \\
& \left.+m\left(\frac{1}{1+n}-\beta(1+n, 1+\alpha)\right)\left[\left|f^{(n)}\left(\frac{a}{m}\right)\right|+\left|f^{(n)}\left(\frac{b}{m}\right)\right|\right]\right\} .
\end{aligned}
$$

So, the proof is done.

Corollary 3.1. In Theorem 3.1., if we elect $\alpha=m=1$, we attain

$$
|\Re| \leq \frac{(1+n)(b-a)^{1+n}}{2^{1+n}(2+n) !}\left\{\left|f^{(n)}(a)\right|+\frac{2}{(1+n)}\left|f^{(n)}\left(\frac{b+a}{2}\right)\right|+\left|f^{(n)}(a)\right|\right\} .
$$

Theorem 3.2. Let $f$ defined from $[0, b]$ to $\mathbb{R}$ be $n$-times differentiable $(\alpha, m)$-convex function. If $f^{(n)} \in L[a, b]$ with $0<a<b$ then

$$
\begin{aligned}
|\Re| \leq \frac{(b-a)^{n+1}}{2^{1+n} n !(1+n p)^{\frac{1}{p}}(1+\alpha)^{\frac{1}{q}}}\{ & \left(\left|f^{(n)}\left(\frac{b+a}{2}\right)\right|^{q}+\alpha m\left|f^{(n)}\left(\frac{a}{m}\right)\right|^{q}\right)^{\frac{1}{q}} \\
& \left.+\left(\left|f^{(n)}\left(\frac{b+a}{2}\right)\right|^{q}+\alpha m\left|f^{(n)}\left(\frac{b}{m}\right)\right|^{q}\right)^{\frac{1}{q}}\right\}
\end{aligned}
$$

where $(\alpha, m) \in[0,1] \times[0,1], n$ naturel number, $n \geq 1$ for $p>1$ and $\frac{1}{p}+\frac{1}{q}=1$.

Proof. Under necessary stipulations of Theorem 3.2., by using features of absolute value 
and Hölder inequality in Lemma 2.1. we note that

$$
\begin{aligned}
& |\Re| \leq \frac{(b-a)^{1+n}}{2^{1+n} n !}\left\{\int_{0}^{1}(1-t)^{n}\left|f^{(n)}\left(t \frac{b+a}{2}+(1-t) a\right)\right| d t\right. \\
& \left.+\int_{0}^{1}(1-t)^{n}\left|f^{(n)}\left(t \frac{b+a}{2}+(1-t) b\right)\right| d t\right\} \\
& \leq \frac{(b-a)^{1+n}}{2^{1+n} n !}\left\{\left(\int_{0}^{1}(1-t)^{n p} d t\right)^{\frac{1}{p}}\left(\int_{0}^{1}\left|f^{(n)}\left(t \frac{b+a}{2}+(1-t) a\right)\right|^{q} d t\right)^{\frac{1}{q}}\right. \\
& \left.+\left(\int_{0}^{1}(1-t)^{n p} d t\right)^{\frac{1}{p}}\left(\int_{0}^{1}\left|f^{(n)}\left(t \frac{b+a}{2}+(1-t) b\right)\right|^{q} d t\right)^{\frac{1}{q}}\right\} .
\end{aligned}
$$

With simple calculation and using $(\alpha, m)$-convexity of $\left|f^{(n)}\right|^{q}$ on $[0, b]$ we note that

$$
\begin{aligned}
|\Re| \leq \frac{(b-a)^{1+n}}{2^{1+n} n !}\left\{\left(\frac{1}{1+n p}\right)^{\frac{1}{p}}\left(\int_{0}^{1}\left(t^{\alpha}\left|f^{(n)}\left(\frac{b+a}{2}\right)\right|^{q}+m\left(1-t^{\alpha}\right)\left|f^{(n)}\left(\frac{a}{m}\right)\right|^{q}\right) d t\right)^{\frac{1}{q}}\right. \\
\left.+\left(\frac{1}{1+n p}\right)^{\frac{1}{p}}\left(\int_{0}^{1}\left(t^{\alpha}\left|f^{(n)}\left(\frac{b+a}{2}\right)\right|^{q}+m\left(1-t^{\alpha}\right)\left|f^{(n)}\left(\frac{b}{m}\right)\right|^{q}\right) d t\right)^{\frac{1}{q}}\right\}(14) \\
=\frac{(b-a)^{1+n}}{2^{1+n} n !}\left\{\left(\frac{1}{1+n p}\right)^{\frac{1}{p}}\left(\frac{1}{1+\alpha}\left|f^{(n)}\left(\frac{b+a}{2}\right)\right|^{q}+m\left(1-\frac{1}{1+\alpha}\right)\left|f^{(n)}\left(\frac{a}{m}\right)\right|^{q}\right)^{\frac{1}{q}}\right. \\
\left.+\left(\frac{1}{1+n p}\right)^{\frac{1}{p}}\left(\frac{1}{1+\alpha}\left|f^{(n)}\left(\frac{b+a}{2}\right)\right|^{q}+m\left(1-\frac{1}{1+\alpha}\right)\left|f^{(n)}\left(\frac{b}{m}\right)\right|^{q}\right)^{\frac{1}{q}}\right\} .
\end{aligned}
$$

With rearranging the last step, the proof is completed.

Corollary 3.2. By electing $\alpha=m=1$ in Theorem 3.2., we handle the inequality for convex functions given below

$$
\begin{aligned}
|\Re| \leq & \frac{(b-a)^{1+n}}{2^{1+\frac{1}{q}+n} n !(1+n p)^{\frac{1}{p}}} \\
& \times\left\{\left(\left|f^{(n)}\left(\frac{b+a}{2}\right)\right|^{q}+\left|f^{(n)}(a)\right|^{q}\right)^{\frac{1}{q}}+\left(\left|f^{(n)}\left(\frac{b+a}{2}\right)\right|^{q}+\left|f^{(n)}(b)\right|^{q}\right)^{\frac{1}{q}}\right\}
\end{aligned}
$$

Theorem 3.3. Let $f$ defined from $[0, b]$ to $\mathbb{R}$ be $n$ - times differentiable $(\alpha, m)$-convex function. If $f^{(n)} \in L[a, b]$ with $0<a<b$ then 


$$
\begin{aligned}
|\Re| \leq & \frac{(b-a)^{1+n}}{2^{1+n} n !(1+n)^{1-\frac{1}{q}}} \\
& \times \sum_{i=a, b}\left(\begin{array}{l}
\beta(1+n, 1+\alpha)\left|f^{(n)}\left(\frac{b+a}{2}\right)\right|^{q} \\
+m\left(\frac{1}{1+n}-\beta(1+n, 1+\alpha)\right)\left|f^{(n)}\left(\frac{i}{m}\right)\right|^{q}
\end{array}\right)^{\frac{1}{q}} .
\end{aligned}
$$

where $(\alpha, m) \in[0,1] \times[0,1], n$ naturel number, $n \geq 1$ and $q$ real number, $q \geq 1$.

Proof. Under the conditions of Theorem 3.3. by using properties of absolute value and Power mean inequality in Lemma 2.1. we handle

$$
\begin{aligned}
& |\Re| \leq \frac{(b-a)^{1+n}}{2^{1+n} n !}\left\{\int_{0}^{1}(1-t)^{n}\left|f^{(n)}\left(t \frac{b+a}{2}+(1-t) a\right)\right| d t\right. \\
& \left.\quad+\int_{0}^{1}(1-t)^{n}\left|f^{(n)}\left(t \frac{b+a}{2}+(1-t) b\right)\right| d t\right\} \\
& \leq \frac{(b-a)^{1+n}}{2^{1+n} n !} \\
& \quad \times \quad\left(\int_{0}^{1}(1-t)^{n} d t\right)^{1-\frac{1}{q}}\left(\int_{0}^{1}(1-t)^{n}\left|f^{(n)}\left(t \frac{b+a}{2}+(1-t) a\right)\right|^{q} d t\right)^{\frac{1}{q}} \\
& \left.\quad+\left(\int_{0}^{1}(1-t)^{n} d t\right)^{1-\frac{1}{q}}\left(\int_{0}^{1}(1-t)^{n}\left|f^{(n)}\left(t \frac{b+a}{2}+(1-t) b\right)\right|^{q} d t\right)^{\frac{1}{q}}\right\}
\end{aligned}
$$

Calculating some expressions and using $\left|f^{(n)}\right|^{q}$ as $(\alpha, m)$-convex function we note that

$$
\begin{aligned}
& |\Re| \leq \frac{(b-a)^{1+n}}{2^{1+n} n !} \\
& \quad \times\left\{\left(\frac{1}{1+n}\right)^{1-\frac{1}{q}}\left(\int_{0}^{1}(1-t)^{n}\left(t^{\alpha}\left|f^{(n)}\left(\frac{b+a}{2}\right)\right|^{q}+m\left(1-t^{\alpha}\right)\left|f^{(n)}\left(\frac{a}{m}\right)\right|^{q}\right) d t\right)^{\frac{1}{q}}\right. \\
& \left.\quad+\left(\frac{1}{1+n}\right)^{1-\frac{1}{q}}\left(\int_{0}^{1}(1-t)^{n}\left(t^{\alpha}\left|f^{(n)}\left(\frac{b+a}{2}\right)\right|^{q}+m\left(1-t^{\alpha}\right)\left|f^{(n)}\left(\frac{b}{m}\right)\right|^{q}\right) d t\right)^{\frac{1}{q}}\right\} \\
& =\frac{(b-a)^{1+n}}{2^{1+n} n !} \times\left\{( \frac { 1 } { 1 + n } ) ^ { 1 - \frac { 1 } { q } } \left(\begin{array}{l}
\beta(1+n, 1+\alpha)\left|f^{(n)}\left(\frac{b+a}{2}\right)\right|^{q} \\
\left.+m\left(\frac{1}{1+n}-\beta(1+n, 1+\alpha)\right)\left|f^{(n)}\left(\frac{a}{m}\right)\right|^{q}\right)^{\frac{1}{q}}
\end{array}\right.\right.
\end{aligned}
$$




$$
\left.+\left(\frac{1}{1+n}\right)^{1-\frac{1}{q}}\left(\begin{array}{l}
\beta(1+n, 1+\alpha)\left|f^{(n)}\left(\frac{b+a}{2}\right)\right|^{q} \\
+m\left(\frac{1}{1+n}-\beta(1+n, 1+\alpha)\right)\left|f^{(n)}\left(\frac{b}{m}\right)\right|^{q}
\end{array}\right)^{\frac{1}{q}}\right\}
$$

With editing the last step, desired result is gathered.

Corollary 3.3. In Theorem 3.3., by electing $\alpha=m=1$, then we achieve

$$
\begin{aligned}
|\Re| \leq \frac{(b-a)^{1+n}}{2^{1+n}(1+n) !}\{[ & {\left[\frac{1}{(2+n)}\left|f^{(n)}\left(\frac{b+a}{2}\right)\right|^{q}+\left(\frac{1+n}{2+n}\right)\left|f^{(n)}(a)\right|\right]^{\frac{1}{q}} } \\
& \left.+\left[\frac{1}{2+n}\left|f^{(n)}\left(\frac{b+a}{2}\right)\right|^{q}+\left(\frac{1+n}{2+n}\right)\left|f^{(n)}(b)\right|\right]^{\frac{1}{q}}\right\} .
\end{aligned}
$$

\section{CONCLUSION}

In conclusion, Hermite-Hadamard type new inequalities related to convex functions have been establihed. Then some new corollaries have been obtained by choosing some particular selections of $\alpha$ and m." Our results include integral form and discrete form of sum of function values together.

\section{REFERENCES}

Alomari MW, Latif MA, Hussain S, 2012. On Ostrowski-type inequalities for functions whose derivatives are convex and convex functions with applications. Tamkang Journal of Mathematics, 43 (4): 521-532.

Bakula MK, Özdemir ME, Pečarič J, 2008. Hadamard Type Inequalities for and Convex Functions. Jour. of Ineq. In Pure and App. Math., Vol. 9 (4), Art. 96, 12 pp.

Chaudhry MA, Qadir A, Rafique M, Zubair SM, 1997. Extension of Euler's beta function. Jour. of Comp. and App. Math., Vol 78, 19-32 pp.

Dragomir SS, Pearce CEM, 2000. Selected Topics on HermiteHadamard Inequalities and Applications. RGMIA Monographs, Victoria University.

Gürbüz, M., 2013. Farklı Türden Konveks Fonksiyonların Çarpımı Üzerine İntegral Eşitsizlikleri ve Uygulamaları. Doktora Tezi, Fen Bilimleri Enstitüsü, Atatürk Üniversitesi, Erzurum.

İşcan İ, 2013. A new generalization of some integral inequalities for convex functions. Mathematical Sciences, 7 (22), 8 pp.

Lara T, Quintero Roy, Rosales E, 2017. Convexity and Functional Equations. Moroccan J. Pure and Appl. Anal., Vol 3(1), 56-62 pp.
Interested readers can find new results by using the aforementioned lemma for other kinds of convex functions or try to get better upper bounds for left side of the lemma by using different properties. Also by applying several convex functions to inequalities proved in this study, some new relations between special means can be found out.

Miheşan VG, 1993. A generalization of the convexity. Seminar on Functional Equations, Approx. and Convex., Cluj-Napoca, Romania.

Özdemir ME, Kavurmacı H, Set E, 2010. Ostrowski's Type Inequalities for Convex Functions. Kyungpook Math. J. 50: 371-378.

Özdemir ME, Avc1 M, Kavurmac1 H, 2011. Hermite-Hadamardtype inequalities via convexity. Comput. Math. Appl., 61: 2614-2620.

Set E, Özdemir ME, Sarıkaya MZ, Karakoç F, 2017. HermiteHadamard type inequalities for convex functions via fractional integrals, Moroccan Journal of Pure and Applied Analysis 3 (1): $15-21$.

Toader G, 1984. Some generalizations of the convexity. Proceedings of The Colloquium On Approximation and Optimization, Univ. Cluj-Napoca, Cluj-Napoca, 329-338.

Yıldız Ç, Gürbüz M, Akdemir AO, 2013. The Hadamard Type Inequalities for Convex Functions. Konuralp Journal of Mathematics, 1 (1): 40-47.

Y1ldız Ç, 2018. New general integral inequalities for convex functions. New Trends in Mathematical Sciences, 6 (1): 1-7. 